\title{
Aprendizajes basados en retos en la formación previa y continua de estudiantes de pedagogía para educación básica alternativa
}

\section{Learning based on challenges in the previous and continuous training of pedagogy students for alternative basic education}

Luis Alberto Rodríguez de los Ríos

Correo: 1rodriguez@une.edu.pe

ORCID: https://orcid.org/0000-0002-2274-695X

Rector de la Universidad Nacional de Educación Enrique Guzmán y Valle, La Cantuta

Héctor Juan Pumayauli Zavaleta

Correo: hpumayauli12@gmail.com

ORCID: https://orcid.org/0000-0002-0772-3901

Consultor de la DVV International

César Hildebrando Delgado Herencia

Correo: cdelgado411122@yahoo.es

ORCID: https://orcid.org/0000-0001-9759-1684

Asesor externo de la DVV International

\begin{abstract}
Resumen
Se presenta el avance de un proyecto de investigación, en su fase de trabajo de gabinete sobre el aprendizaje basado en retos (ABR) a experimentarse con estudiantes de un programa de formación universitaria en educación básica alternativa (EBA), asimismo en talleres sobre de ciudadanía plena en docentes de la modalidad en servicio. La pedagogía como ciencia de la educación acoge de otras disciplinas prácticas exitosas, una es la técnica de $\mathrm{ABR}$, que cambia las sesiones de aprendizajes frentistas actualmente dominantes en EBA. Como tal, puede aplicarse a diversas situaciones educativas en las instituciones, para el caso al proceso de enseñanza/aprendizaje de formación profesional previa y continua de docentes para esa modalidad. La idea principal de la técnica, los estudiantes construyan un reto/problema relevante en la comunidad, avanzan a su investigación y comunican los resultados mediante medios digitales, así como utilizan los
\end{abstract}

Contacto: revistaeduca@umch.edu.pe_ISSN: 2617-0337 revistas.umch.edu.pe/ 
protocolos para futuras aplicaciones, esto se logra en las jornadas de cuatro fases: el reto, la investigación, los resultados y la comunicación. La técnica tiene bondades como limitaciones.

Palabras clave: Aprendizaje experiencial. Pedagogía. Problema. Reto. Investigación. Respuestas. Educación Básica Alternativa.

\section{Summary}

The progress of a research project is presented, in its phase of cabinet work on learning based on challenges to be experienced with students from a university training program in alternative basic education.Pedagogy as a science of education welcomes other successful practical disciplines, one is the challenge-based learning technique-CBL-, that changes the currently dominant frontist learning sessions in ABE. As such, it can be applied to various educational situations in institutions, in the case of the teaching / learning process of previous and continuous professional training of teachers for this modality. The main idea of the technique, the students build a relevant challenge / problem in the community, advance their research and communicate the results through digital means, as well as use the protocols for future applications, this is achieved in the four-phase sessions: the challenge, research, results and communication. The technique has benefits as limitations.

Keywords: Experiencial learning. Pedagogy. Issue. Challenge. Investigation. Answers. Alternative Basic Education.

\section{Introducción}

Desde inicio del presente siglo, los sistemas educativos universitarios a nivel mundial y especialmente, los de Latinoamérica, han venido experimentando cambios cualitativos en su cultura institucional, en sus procesos académicos, administrativos y de gestión. Siendo los más significativos los referidos a sus estructuras curriculares y a las formas de enseñanza-aprendizaje o metodologías didácticas; apostando por un servicio educativo de calidad, equitativo, pertinente e inclusivo (Tünnermann [2006]).

Situación, que ha generado un cambio educativo paradigmático centrado en el estudiante y en los procesos de construcción de sus diversos saberes personales y académicoprofesionales. Vale decir, por un aprendiz metacognitivo, estratégico, autónomo y competente (Pozo y Monereo, 1999; Tejeda y Sánchez, 2012), concordante con la sociedad de la información y el conocimiento que está inserto; "Buscando con ello superar las metodologías tradicionales orientadas hacia la memorización, la acumulación y la repetición mecánica de datos para privilegiar el saber con la información y la resolución de problemas con sentido para los estudiantes" (Tobón, 2006, p.2).

El mencionado cambio paradigmático se ha visto fortalecido y transformado con la omnipresencia e incorporación de las tecnologías digitales mediante la configuración de 
entornos virtuales y personales de aprendizaje y enseñanza (Coll y Monereo, 2008; Barroso y Cabero, 2013; Cabero, 2014; Zambrano, 2012), optimizando y expandiendo los procesos inherentes y la autogestión del conocimiento disciplinar y formativo. En este sentido, Negroponte (2008) citado por Zambrano (2012), sostiene "La tecnología deja de ser solo un asunto técnico para procesarse y pensarse en el contexto de una labor de enseñanza-aprendizaje y de una formación integral del estudiante..." (p.82), institucionalizándose la educación o docencia virtual que, en los últimos meses ha adquirido una vigencia plena y sostenida en forma acelerada en la actual pandemia producida por el Covid-19.

En ese contexto, han resurgido y brotado nuevas técnicas didácticas de naturaleza activa e interactiva, derivadas de la concepción teórica del aprendizaje experiencial, como el aprendizaje basado en problemas, el estudio de casos, el aprendizaje basado en la investigación, el aprendizaje colaborativo y el aprendizaje basado en retos, entre otros. Los cuales centran su atención en un estudiante que asume responsabilidades y resulta protagonista de su propio aprendizaje, de su formación y desarrollo competencial.

Uno de los sistematizadores y mentores del modelo de aprendizaje experiencial fue Dewey (1938), citado por Baena (2009) quien postulaba que toda educación verdadera se realiza mediante la experiencia, vinculando la reflexión y la compresión con la acción. Sosteniendo que no es posible saber y comprender completamente sin hacer. Ubicándose en esa línea de pensamiento a Lewin, Piaget, y Kilpatrick, Rogers y Kolb. Siendo Kolb (1984), citado por Alonso Gallego, Honey (1997), uno de los autores que más ha analizado y profundizado en el efecto del aprendizaje experiencial en la adquisición de conocimiento. Asegurando que el conocimiento se construye mediante la transformación de la experiencia.

Por tanto, el aprendizaje basado en retos es una técnica didáctica que involucra activamente al estudiante en una situación problemática real, relevante y de vinculación sobre el entorno, lo cual implica la definición de un desafío y la implantación de una solución (Delgado, 2018; Fidalgo, 2017; García, 2019; Instituto Tecnológico de Monterrey, 2018). Permitiendo que los estudiantes se comprometan desde sus talentos individuales, sus sueños, sus gustos y el trabajo colaborativo. Originalmente fue introducida por Apple para su uso en la educación K-12 (nivel medio superior), extendiéndose posteriormente a la educación superior. Entendiéndose como, reto "a una experiencia vivencial diseñada para exponer al alumno a una situación desafiante del entorno para lograr objetivos especificos de aprendizaje". (Instituto Tecnológico de Monterrey, 2018).

Se trata de un modelo estructurado del curso que tiene una base en las estrategias metodológicas inductivas. En vez de presentar a los estudiantes un problema para resolver, el ABR ofrece conceptos generales de los que los estudiantes obtienen los retos que tendrán que abordar. Además, el ABR fomenta el uso de las tecnologías web y móviles, tales como herramientas y wikis colaborativos, que están disponibles para los 
estudiantes, pero que no se utilizan en las asignaturas (Delgado, Rojo, Torres y Becerril, 2018).

Es un modelo de enfoque, preferentemente interdisciplinar, y promueve proyectos que involucran a la comunidad en general. Combinación que permite a los estudiantes elegir su desafío y la vinculación de esos desafíos a la interacción de la comunidad, aumenta los esfuerzos y la acción de los estudiantes en un resultado eficaz y productivo.

\section{Marco de la técnica del ABR}

La técnica fue desarrollada en las ciencias de la ingeniería a principios del presente siglo. Se caracteriza por retos o desafíos que tienen en su núcleo un gran problema o problemas o conflictos comunales que impelen a resolverlos desde la mirada provocadora que significan. Si hay un problema, entonces requiere estudio desde la perspectiva de la pedagogía. Como se sabe, el problema o problemas son constructos que tienen solución o soluciones, o respuesta o respuestas, no son imposibles.

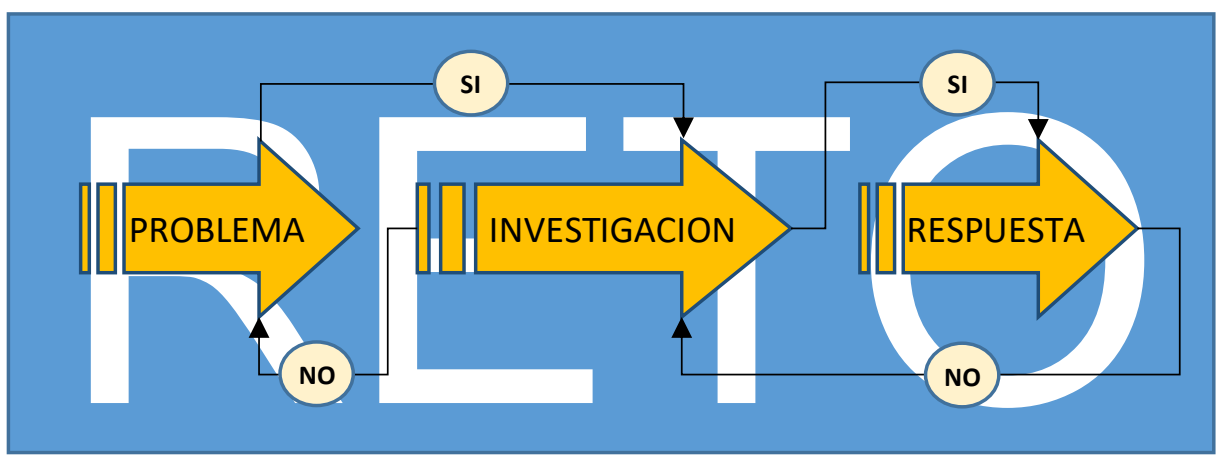

Figura1: Elaboracion propia

Tres grandes puertos tienen la técnica como se observa en el diagrama, puede ser aplicado a diversas disciplinas, no solo a las ingenierías que buscan "soluciones"; en la pedagogía se averiguan "respuestas". Ante el problema/reto se investiga las posibles repuestas pasando por cada uno de esos momentos, si no son satisfactorios, se retorna hasta lograr el propósito. Si no es posible investigar es porque el reto/problema no es lo suficiente potente para hacerlo; al igual si no se obtienen las respuestas al reto inicial entonces se debe seguir investigando. Es probable que las respuestas no sean del todo satisfactorias, lo que abre discursos constantes hacia la búsqueda de la verdad.

De ese modo, un rasgo general del ABR es la flexibilidad, aunada a lo holístico, dado que demanda una mirada multidisciplinar; desde la pedagogía estas dos dimensiones contienen rasgos particulares dado que como técnica es afín a otras como la vivencial,

Contacto: revistaeduca@umch.edu.pe_ISSN: 2617-0337 revistas.umch.edu.pe/ 
colaborativa, la activa, de proyectos. Hoy enormemente facilitadas por las herramientas digitales. Por ello, es una técnica amable con la educación a distancia, la pedagogía invertida, el trabajo horizontal entre maestros y estudiantes/participantes.

\section{Modelo general ABR en pedagogía}

El diagrama muestra las cuatro etapas generales del modelo ABR desde la mirada de la pedagogía, en síntesis: la fase 1, el reto; la fase 2, la investigación; la fase 3, los resultados, y, la fase 4 , la comunicación. Los efectos de la técnica sirven de insumos para realimentación. También es necesario hacer una presentación del dispositivo pedagógico a los participantes de modo que las fases puedan ser entendidas y rápidamente realizadas.

\section{Fase 1. El reto}

La fase 1 del reto tiene cuatro momentos. La idea general consiste en la definición del problema que es a su vez un desafío de cuestiones esenciales, todos los participantes tienen que identificar con claridad el problema en la vida real con la ayuda del docente facilitador, esto lleva al análisis que produce actividades que son los componentes del reto/problema, a su vez son contrastados con los saberes previos de todos. En este momento no se maneja más información y conocimiento que los que tienen los integrantes en su memoria asociativa.

Todo eso debería conducir a contar con un reto general y algunos específicos en sus rasgos principales que desencadenen motivaciones internas expresadas en actividades, son estas que implican la formación de sub grupos por cada desafío especifico derivado del reto general. Es recomendable que los sub grupos no pasen de cinco participantes y cuenten con un coordinador, de este modo quedan fijadas las responsabilidades dado que todo el trabajo es grupal, colaborativo, activo; los tiempos a usar y las reglas que guiarán toda la técnica están inmersas en este momento.

Contacto: revistaeduca@umch.edu.pe_ISSN: 2617-0337 revistas.umch.edu.pe/ 


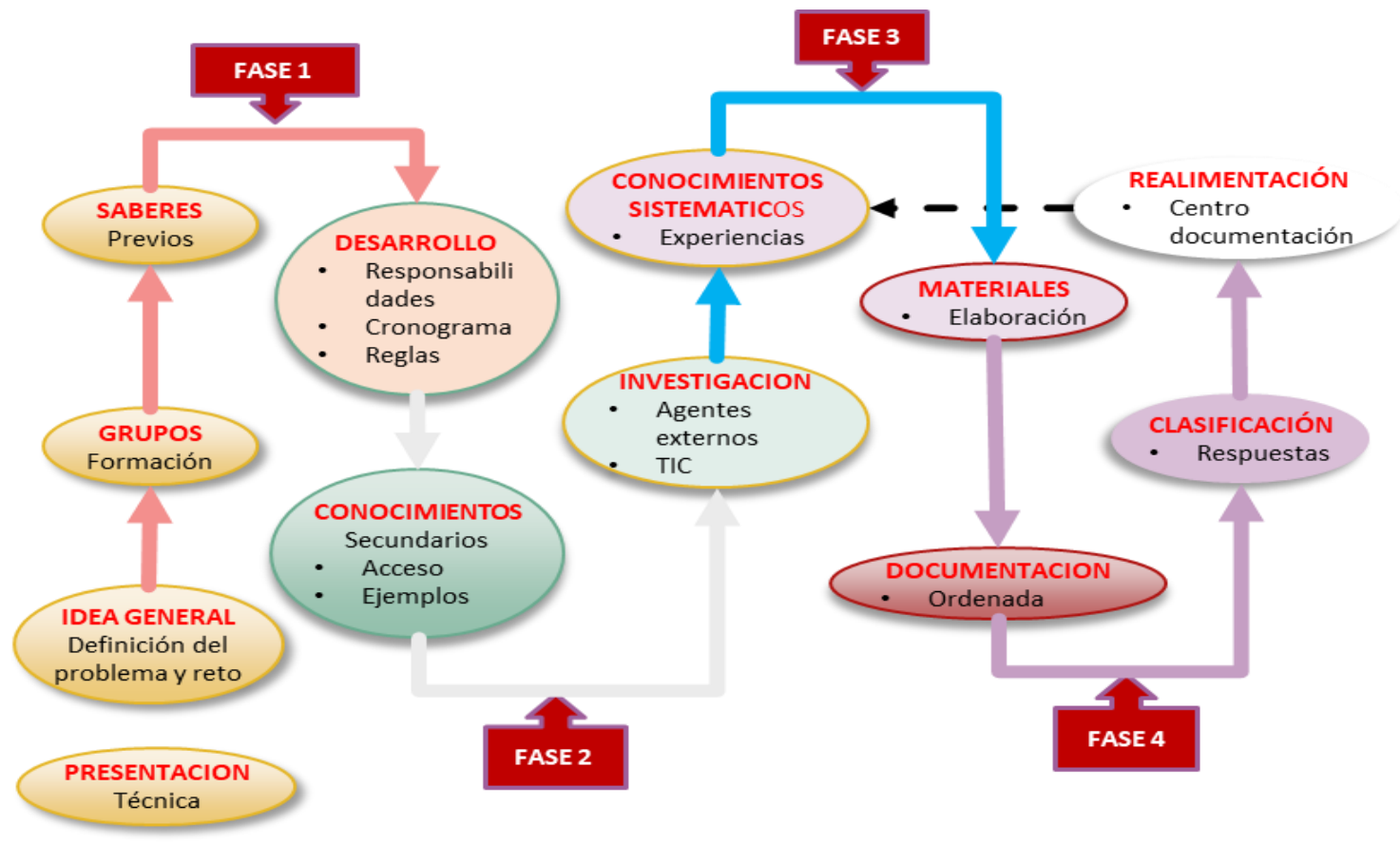

Figura 2: Elaboración propia

En cada una de las sub fases es necesario que el equipo tenga un portafolio para ir acumulando los trabajos realizados (resúmenes, videos, hallazgos, etc.) que van a ser utilizados a lo largo del ABR.

\section{Fase 2. La investigación}

Esta fase implica desarrollar las tareas en los equipos previstas por las actividades; están centradas en el registro de información "secundaria" sobre cada uno de los retos específicos. El asunto central es: lecturas de primera mano de referencias como diarios, revistas no especializadas, páginas WEB, redes sociales, todo ello lleva a efectuar resúmenes (las conocidas fichas) de modo de ir profundizando desde esos abordajes los retos.

Con toda esa inicial inmersión se pasa a la sistematización de información de mayor nivel, de especialistas en revistas de preferencia indexadas, conferencias, incluso consultas personales a expertos sobre los contenidos del reto específico. Todo esto lleva a tener información y conocimiento sistematizado sobre el problema/reto que sirve de apoyo a la imaginación de conjeturas, suposiciones, presunciones de las posibles respuestas como de problemas colaterales.

Esas conjeturas en la medida que estén sustentadas en mayor información y conocimiento deberían llevar a razonables hipótesis de trabajo sobre el tema en cuestión,

Contacto: revistaeduca@umch.edu.pe_ISSN: 2617-0337 revistas.umch.edu.pe/ 
donde las respuestas al reto ya dejan de ser meras presunciones dado que es viable poder identificar variables e indicadores todavía muy gruesos.

Igual que la fase anterior, todo lo realizado va siendo ubicado en el portafolio de cada sub grupo. Así las hipótesis de trabajo, las variables dependientes e indicadores de cada uno de los retos específicos.

\section{Fase 3. Los resultados}

Todo lo avanzado en el ABR debe llevar a los equipos a la contrastación de la hipótesis de trabajo, esto es, confrontar la variable independiente y su indicador con la realidad del conocimiento sistemático y si es posible contar con algún especialista sobre el tema en cuestión que pueda apoyarla, aquí retoma un rol importante el docente facilitador que va conduciendo o mediando - mejor- entre el reto, la información sistematizada, de modo de coadyuvar a que los equipos vayan generando sus propias puestas en común en consensos sobre aquellas hipótesis.

Un aspecto importante es que el despeje de las hipótesis de los retos específicos debe llevar a dar respuestas al reto general, que es el gran desafío de la técnica pedagógica. Aquí ya el grupo ABR roza conclusiones del reto general, que sean viables, razonadas, producto de investigación y que va dejando en todos los participantes la satisfacción de haber construido conocimiento desde un constructo que se inició como problema se convirtió en desafío y ahora está empoderado con respuestas derivadas de las conclusiones generales y específicas que conforman un cuerpo orgánico de explicaciones. Es la etapa que mayor tiempo consume al ABR, y todo lo debatido -que implica posiciones- debe ser acumulado en el portafolio.

\section{Fase 4. La comunicación}

Con la apropiación de las conclusiones se procede a imaginar cómo comunicar las respuestas. Esto implica acudir a los portafolios y utilizar los medios más eficaces para ello. De un lado, los hallazgos que pueden tener el tinte de originales o de rescate, pero siempre todos importantes tanto para el progreso del conocimiento como para los auto aprendizajes de los participantes y las auto enseñanzas de los facilitadores. Las herramientas digitales por su vistosidad y amabilidad son buenas cajas de opciones, videos, blog, páginas web, etc. Pero también los otros medios como cartillas, informes, folletos, artículos, etc.

Contacto: revistaeduca@umch.edu.pe_ISSN: 2617-0337 revistas.umch.edu.pe/ 
La comunicación de los resultados es también significativa, se utiliza el camino inverso de la técnica, se clasifica las respuestas por orden de importancia e impacto, se recopila todo lo actuado con el propósito que sirvan de insumos a futuras experiencias con ABR. Y se envían a un centro de documentación en la idea del conocimiento discursivo de la ciencia.

4. Bondades del ABR. De las experiencias que se tienen registradas en sus años de práctica el ABR (Bolaños s/f; Garza, 2016) muestra los siguientes beneficios donde el estudiante:

- Practica con agudeza de temas relevantes para su vida y de la comunidad, en la medida que los problematiza antes de tener respuestas firmes y las verbaliza como desafíos o retos.

- Concientiza desde el problema/reto y asume el duro camino de la investigación, imagina enfoques, labora en equipo y acude a otras disciplinas.

- Participa plenamente desde la construcción del problema/reto y en todas las fases siempre está implicado, de modo que la técnica lo conduce a respuestas razonadas y no meras opiniones.

- Comunica de manera pertinente usando los medios de información de todo tipo para lo cual desarrolla habilidades cognitivas, procedimentales y de inserción social.

5. Restricciones del ABR. Ninguna técnica pedagógica es perfecta y ABR tiene sus limitaciones, entre las principales están:

- No puede ser utilizada en toda la malla curricular tradicional; las asignaturas deberían ser diseñadas en proyectos o específicamente para usar el ABR.

- Que el reto sea tan amplio, global, que no permita imaginar desafíos específicos, aquello equivale a un problema enorme, es decir imposible.

- Puede sesgarse a que los participantes descuiden los aprendizajes del reto y se centren en pruebas.

- Cuando se aplica el ABR a varias asignaturas, las respuestas generales de todas solo se pueden tener al finalizar el ciclo.

Contacto: revistaeduca@umch.edu.pe_ISSN: 2617-0337 revistas.umch.edu.pe/ 
6. Presentación del ABR a los participantes. El modelo general debe ser comprendido en su cabalidad por todos los participantes que desconozcan el ABR. Es necesario dedicar una sesión exclusiva para su aprendizaje/enseñanza. La técnica se reduce a indagar soluciones a un problema principal convertido en un desafío personal y grupal, por ejemplo, la corrupción ciudadana.

Se trata además que los participantes desarrollen sus capacidades que lleven a lograr la competencia, verbigracia, "concientización de la corrupción ciudadana" y las consecuencias para vivir bien en una sociedad para todos y enfilada hacia el bien y hogar comunes. El diagrama describe con claridad para que no queden dudas en los participantes de las bondades -así como sus restricciones- de la técnica pedagógica. El mejor camino metodológico es el diálogo con los estudiantes, para ello se recomienda usar diagramas, videos, recortes de diarios, etc.

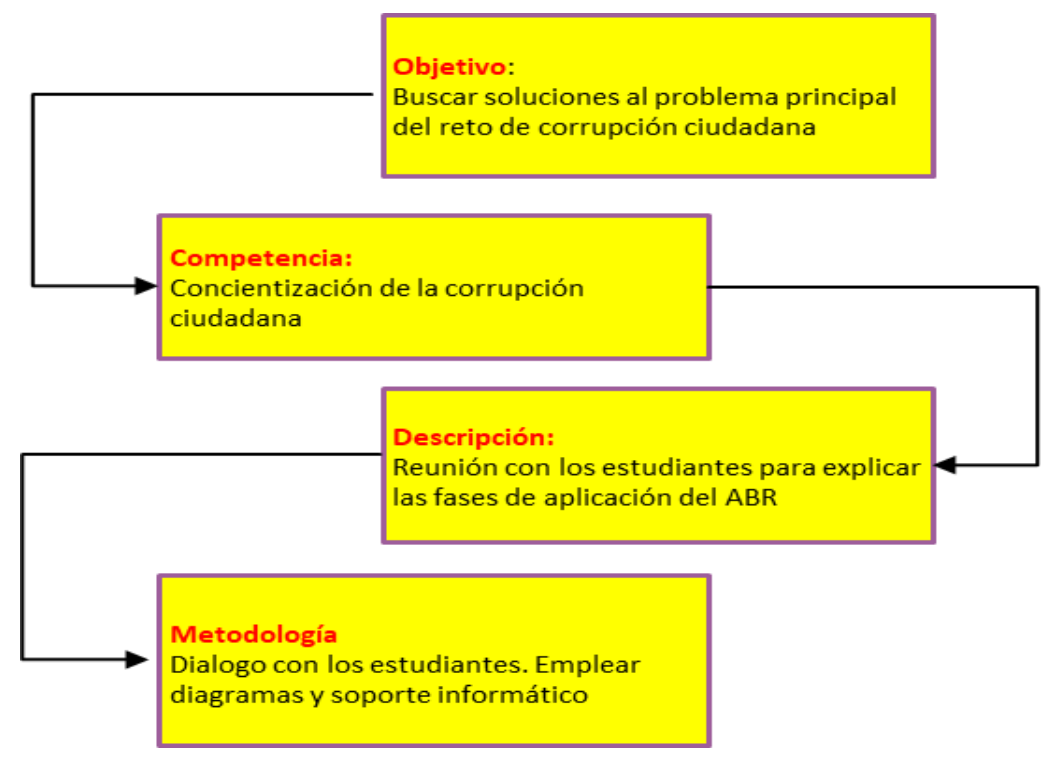

Figura 3: Elaboración propia

\section{La educación básica alternativa (EBA)}

La educación como derecho humano esencial no está garantizada en toda la compleja demanda de la ciudadanía peruana; la formación escolar en educación básica que es obligatoria y gratuita cuando la brinda el Estado en sus instituciones educativas, se estima en más de siete millones los peruanos - principalmente peruanas- que no la han concluido. En particular, cuando se refiere a la que jóvenes y adultos que por 
razones socioeconómicas deben abandonar sus trayectorias escolares en aras de contribuir a la subsistencia de sus familias.

A esa población es para quien va destinada lo que en el país se nomina "educación básica alternativa”, diseñada en la Ley General de Educación de 2003. En los 17 años de creada como modalidad del sistema educativo nacional ha pasado por cuatro administraciones presidenciales y cerca a dieciséis ministros de educación y sigue con una matrícula que llega ajustadamente al 3\% de la población escolar en las escuelas estatales. Su rasgo principal es su invisibilidad para innovaciones no solo en gestión sino significativamente en pedagogía para esos grupos etarios mayores de quince años.

Actualmente, entre sus nudos críticos en pedagogía para jóvenes y adultos están la "debilidad en procesos pedagógicos para la modalidad", "desarticulación entre la educación formal y no formal" con repercusiones en la auto formación integral de sus estudiantes, dado que la modalidad no logra identidad respecto a la educación de niños y adolescentes, y como prestación de servicio no impulsa los procesos autónomos de aprendizajes en los estudiantes, habiendo retornado a prácticas frentistas de lo que fue la extinta "educación de adultos" del siglo anterior.

Afrontar esa lacerante realidad necesita de la aplicación de nuevas técnicas pedagógicas más amables, pero también provocadoras por parte de los profesionales en la educación para esa modalidad. Creemos que la técnica pedagógica de auto aprendizajes basado en retos es una herramienta que los docentes pueden adecuar en los heterogéneos grupos/clase en sus sesiones de aprendizajes, así como en las educaciones no formales para jóvenes y adultos. Y, esta renovación de la pedagogía para jóvenes y adultos se empieza en la formación previa y continua de sus docentes.

La Universidad Nacional de Educación Enrique Guzmán y Valle (UNE), como única entidad universitaria de formación previa y continua de profesionales para la EBA en el país, en su renovación curricular desea introducir el dispositivo pedagógico del $\mathrm{ABR}$ en la formación de los futuros docentes de la modalidad y que por efecto cascada llegue a estudiantes en los centros educativos de educación básica alternativa (CEBA). Por lo que está empeñada en aplicaciones en las asignaturas actuales que se acojan en la intencionalidad pedagógica en la forma de atención semipresencial (híbrida), así como en eventos con docentes en servicio desde el tema de concientización como reto principal del proceso de enseñanza/aprendizaje, labor que cuenta con cooperación internacional.

\section{Conclusiones}

Contacto: revistaeduca@umch.edu.pe_ISSN: 2617-0337 revistas.umch.edu.pe/ 
La renovación pedagógica de la educación básica de jóvenes y adultos es una necesidad que se ha acrecentado por los acontecimientos actuales y que están afectando a los sistemas educativos. Demanda presente desde la propia ley general de educación que impulsa como forma de atención principal la semipresencialidad en esta Otra Educación. Las tradicionales sesiones de aprendizajes presenciales, deben dar paso a otras formas de pedagogías mucho más amables con los procesos autónomos de aprendizajes de los estudiantes y donde el docente es un mediador/facilitador de los propios conocimientos que los estudiantes van descubriendo como consecuencia de su praxis.

Así, la técnica pedagógica de aprendizajes basado en retos es convergente de otros dispositivos pedagógicos que están inscritos en aquellos auto aprendizajes y que va unida al uso de los medios digitales para que sean los propios estudiantes quienes construyan sus aprendizajes y donde el docente facilitador es el dialogante en las fases de esa técnica e ir forjando los conocimientos necesarios a problemas que los propios estudiantes han construido, es una secuencia virtuosa de cursiva de saberes más retadores y por tanto más inclusivos.

Para ello la UNE está desarrollando pilotos tanto con los estudiantes en formación previa y continua para docentes en la modalidad como con docentes en servicio de manera de contribuir a renovar la pedagogía para jóvenes y adultos en el país. Esto es, a su vez, el $A B R$ resulta un desafío para la universidad en sus dieciocho años de experiencia que tiene formando profesionales de calidad con equidad, que respondan a nuestros propios problemas en el modelo cantuta de formación integral.

\section{Referencias}

Alonso, C., Gallego, D., Honey, P. (1997). Los estilos de aprendizaje. Procedimiento de diagnóstico y mejora. $3^{\mathrm{a}}$ ed. Bilbao: Mensajero.

Baena, V. (Edit.) (2019). El aprendizaje experiencial como metodología docente. Madrid: Narcea. S.A.

Barros, J. y Cavero, J. (Coord.) (2008). Nuevos escenarios digitales. Las tecnologías de la información y la comunicación aplicadas a la formación y desarrollo curricular. Madrid: Pirámide.

Bolaños Oscar (s/f), Aprendizajes basado en retos, Centro de recursos para el aprendizaje. México; Universidad ICESI.

Contacto: revistaeduca@umch.edu.pe_ISSN: 2617-0337 revistas.umch.edu.pe/ 
Cabero, J. (2014). Los entornos personales de aprendizaje (PLE). Málaga: ic Editorial

Coll, C. y Monereo, C. (Eds) (2008). Psicología de la Educación virtual. Madrid: Morata, S.A.

Delgado, G.; Rojo, M.; Torres, J.G. y Becerril, H. (2018). Aprendizaje basado en retos. Revista electrónica Anfei digital. Año 5, № 9, Jul. - Dic.

Fidalgo, A. (2017). Aprendizaje Basado en Retos en una asignatura académica universitaria, Revista Iberoamericana de Informática Educativa, Madrid

García, W. J. (2019). Aprendizaje basado en retos para la solución de problemas con tecnología con mediación TIC para el grado 11 de la I.E. Liceo Gabriela Mistral, Municipio de la Virginia. Tesis de maestría en Informática aplicada a la educación. Universidad Cooperativa de Colombia sede Pereira.

Garza, E. (2016). Aprendizaje basado en retos. México: Instituto Tecnológico y de Estudios Superiores de Monterrey.

Instituto Tecnológico de Monterrey. (2018). Modelo Educativo TEC 21.

Jaimez, C. (2016). Estrategias didácticas en educación superior basadas en aprendizajes. México: Universidad Autónoma Metropolitana de Cuajimalpa.

Monereo, C. y Pozo, J.I. (2003). La universidad ante la nueva cultura educativa. Enseñar y aprender para la autonomía. Madrid: Síntesis, S.A.

Moore, D. (2013). For interns, experience isn't always the best teacher. The Chronicle of Higher Education. Retrieved from: http://chronicle.com/article/For-InternsExperience-Isnt/143073/

Pozo, J. I y Monereo, C. (coord.), (1999). El aprendizaje estratégico: enseñar a aprender desde el currículo. Madrid: Santillana.

Tejeda, R. y Sánchez, P. (2012). Formación basada en competencias profesionales en contextos universitarios. Manta: Mar abierto.

Tobón, S. (2008). Competencias en la Educación Superior. Políticas hacia la calidad. Bogotá: Ecoe Ediciones.

Tünnermann, C. (2006). Pertinencia y calidad de la educación superior. Lección inaugural. Guatemala. Consultado el 10 de noviembre del 2020 en: http://biblio2.url.edu.gt:8991/libros/leccion\%20inaugural2006texto.pdf

Contacto: revistaeduca@umch.edu.pe_ISSN: 2617-0337 revistas.umch.edu.pe/ 
Educa UMCH. Revista sobre Educación y Sociedad, 2020, 16(1), 5-22.

https://doi.org/10.15366/Educa UMCH2019.17.3.001

Zambrano, W, (2012). Modelo de aprendizaje virtual para la educación superior. MAVES basado en tecnologías web 3.0. Bogotá: Ecoe Ediciones. 